\title{
The production of ultra-high purity single isotopes or tailored isotope mixtures by ICP-MS
}

\author{
M. Liezers ${ }^{\mathrm{a}, *}$, O.T. Farmer III ${ }^{\mathrm{a}}$, M.P. Dion ${ }^{\mathrm{a}}$, M.L. Thomas ${ }^{\mathrm{a}}$, G.C. Eiden $^{\mathrm{a}}$ \\ ${ }^{a}$ Pacific Northwest National Laboratory, P.O. Box 999, Richland, WA, 99352, USA
}

\begin{abstract}
We report the development and testing of a collector arrangement for quadrupole Inductively Coupled Plasma Mass Spectrometry (ICP-MS) that for the first time has been used to isolate small quantities of highly enriched $(>99.99 \%)$ single isotopes, with deposition rates $>10 \mathrm{ng} \mathrm{hr}^{-1}$. The collector assembly replaces the standard instrument detector allowing for implantation with simultaneous monitoring of the incident ion current. Even under zero bias implant conditions, low energy $(<10 \mathrm{eV})$ ion collection efficiency was observed to be very high $99 \%$. ${ }^{151} \mathrm{Eu}$ ion currents of $0.1-0.5 \mathrm{nA}$ were collected on a simple, planar foil without resorting to any type of cup configuration. Recovery of the enriched isotope from such foils is much simpler than from a more complex cup configuration. High rejection of adjacent mass isotopes was demonstrated by selectively implanting ${ }^{167} \mathrm{Er}$, then using laser ablation ICP-MS imaging to confirm the absence of any co-implanted ${ }^{166} \mathrm{Er}$ or ${ }^{168} \mathrm{Er}$. The important analytical possibilities of this new approach to isotope ratio measurement, tracer purification, and radiation measurements are discussed.
\end{abstract}

Keywords: Isotope Enrichment; ICP-MS; Ion Implantation; Isotope Dilution; Laser Ablation

\section{Introduction}

Isotope enrichment by mass spectrometry is an old technique proposed as early as the $1920 \mathrm{~s}$, but only with the practical isolation of ${ }^{235} \mathrm{U}$ from ${ }^{238} \mathrm{U}$ by

\footnotetext{
*Corresponding author

Email address: martin.liezers@pnnl.gov (M. Liezers)
}

Preprint submitted to International Journal of Mass Spectrometry October 24, 2014 
Nier et al. was the method potential realized [1]. Their subsequent demonstration that ${ }^{235} \mathrm{U}$ was the principal fissionable isotope of uranium, led to the mass spectrometry being adopted on a massive scale for the Manhattan Project [2]. In the following decades, mass spectrometry was entirely displaced for bulk $\mathrm{U}$ enrichment in favor of more efficient gaseous diffusion [3] and gas centrifuge [4] technologies, but has remained in use on a small scale using Calutron type instruments for the production of enriched isotopes of other elements excluding $\mathrm{U}$ on the $\mathrm{mg}-\mathrm{g}-\mathrm{kg}$ scale. Relatively few of these instruments are in routine operation so access to the isotopically enriched product of this type of mass spectrometer (MS) is restricted; principally owing to their large size and the limited market for enriched isotopes that remains mostly research based $[5,6]$. To produce single isotopes on the gram scale requires the use of high ion currents, typically $\mu \mathrm{A}-\mathrm{mA}$, and relatively high operating pressures, $\approx 1 \times 10^{-5}$ mbar. For magnetic sector-based instruments these conditions lead to low abundance sensitivity and limited isotopic purity - rarely $>99.5 \%$. While these large instruments can produce enriched material at the gram level, most analytical MS measurements performed using enriched isotopes and tracers display detection limits of femtograms to picograms, therefore the presence of other unwanted isotopes of that element in the enriched spike at even nanogram to picogram levels can become a severe hindrance. In addition, the range of enriched isotopes available and their purities are fairly restricted and the cost of tailoring isotopic composition of an element for some specific application is usually cost prohibitive. In recent years a small number of publications have appeared applying the same approach to selectively implanting organic molecules by mass spectrometry $[7,8]$, although preserving their structure and chemical functionality has remained problematic.

In $1996 \mathrm{Hu}$ and Houk [9] reported the deposition rates of the monoisotopic element ${ }^{165} \mathrm{Ho}$ onto a graphite target using ICP-MS. They performed a series of ${ }^{165} \mathrm{Ho}$ depositions at different biases (-10 to $\left.-4500 \mathrm{~V}\right)$ applied to the graphite target, observing significant deposition rates ranging from $2 \times 10^{12}-$ $5 \times 10^{12}{ }^{165} \mathrm{Ho}$ ions s $\mathrm{s}^{-1}$. This equates to depostion rates of up to $1.4 \mathrm{ng} \mathrm{s}^{-1}$ or nearly $5 \mu \mathrm{g} \mathrm{hr}^{-1}$, obtained using an input ${ }^{165} \mathrm{Ho}$ solution concentration of $1000 \mathrm{ppm}$. Variations in the ion deposition rate as a function of the ion kinetic energy were observed and interpreted as changes in the ion stopping mechanism in the shift from low to high ion kinetic energies. They mention the idea of creating altered isotopic layers, but no evalution of isotopic purity achieved by this approach was subsequently reported and the concept of using 
ICP-MS to create isotopically enriched materials has so far not been actively developed.

ICP-MS in its many forms has been widely adopted for both elemental and isotopic analysis with combined ion generation and detection efficiencies currently reaching up to $2-3 \%$ for some large magnetic sector instruments under low signal conditions $[10,11]$. However, this level of performance is probably not sustainable for mass separated ion currents much above $10 \mathrm{nA}$. For quadrupole ICP-MS the combined ion generation and detection efficiency is usually much lower, $<0.01 \%$, but this still equates to incident single isotope ion currents after the quadrupole of $\approx 200 \mathrm{pA}$ or higher using a solution containing $10 \mathrm{ppm}$ of the element where the target isotope displays a modest abundance (25\% or higher). While higher elemental concentrations can be introduced into the ICP-MS, nonlinearity will often result due to space-charge limitations occurring within the plasma sampling interface and ion optics. As the bulk of the total ion current $(\approx 10 \mu \mathrm{A})[12]$ in these regions is carried by the primary plasma gas ion $\left(\mathrm{Ar}^{+}\right)$, argon based molecular ions and various molecular ion combinations of $\mathrm{H}, \mathrm{C}, \mathrm{N}$ and $\mathrm{O}$, these ultimately limit the maximum rate at which an enriched isotope can be accumulated using this approach. The atmospheric ICP ion source is very robust and can turn most elements in the periodic table into positive ions quite efficiently from either solid or liquid aerosols. As a result of this sample introduction flexibility and high sensitivity, ICP-MS instruments have become very widely available.

For the most common quadrupole based units the superior abundance sensitivity of the mass filter alone suggests they can achieve far higher levels of isotopic purity than Calutrons or gaseous diffusion techniques if $n g-\mu \mathrm{g}$ quantities of these highly enriched materials is adequate for the target application. While the production of such small quantities is not without potential isotopic contamination challenges, the amounts of enriched material actually required for tracer spikes in most analytical inorganic mass spectrometry methods can range from pg to $\mu \mathrm{g}$, well within practical reach of the ICP-MS production method proposed in this paper. Typical adjacent mass abundance sensitivity specifications for a quadrupole ICP-MS based on 0.8 amu peak width is $<1 \mathrm{ppm}$ on the low mass side of the peak and $<0.5 \mathrm{ppm}$ on the high mass side. Expressed another way $<1$ cps would be detected at M-1 when the count rate on mass $M$ is $1 \times 10^{6} \mathrm{cps}$ and $<0.5$ cps at $M+1$. Abundance sensitivity at masses \pm 2 amu or further away are extremely difficult to measure but should be $<<0.01 \mathrm{ppm}$ and, unlike magnetic sector mass analyzers, quadrupole mass filters display no significant degradation in abundance 
sensitivity even at relatively high operating pressures up to $5 \times 10^{-5}$ mbar.

In this paper we describe the design of an isotope collection assembly for a quadrupole ICP-MS and results obtained in the production of nanogram quantities of highly enriched $(\approx 99.99 \%){ }^{151} \mathrm{Eu}$ isotope from a diluted $\mathrm{Eu}$ standard with a natural Eu isotopic distribution. The results include: incident ion current measurement, implant efficiency, isotopic purity, and (Laser Ablation) LA-ICP-MS imaging of the ${ }^{151} \mathrm{Eu}$ distribution on a copper foil substrate. The adjacent mass rejection of ${ }^{166} \mathrm{Er}$ and ${ }^{168} \mathrm{Er}$ from ${ }^{167} \mathrm{Er}$ is also demonstrated and the exciting new potential applications of this bench top approach of isotope enrichment are discussed.

\section{Implant Design}

The work we describe was performed using a model PQ-Excell (VG Elemental Ltd, Winsford, England) quadrupole ICP-MS with no major modifications made to the ICP ion source, plasma-sampling interface, primary ion optics, and quadrupole mass filter. The instrument employed a simultaneous pulse counting/analog multiplier model AF-214 (SGE Inc., Austin, TX, USA) and the initial enriched isotope collector design made to mimic the basic shape and the exact length of the electron multiplier to allow for easy substitution. The main body of the collector was machined from PEEK (PolyEther Ether Ketone) with a simple hollow copper cup attached to the end. The $11.0 \mathrm{~mm}$ aperture of the copper cup was set to match the grounded front aperture of the multiplier with a cup depth of $20.0 \mathrm{~mm}$. While the shape of the collector resembles a Faraday cup, no suppressor grid or other electrodes were employed in front of the cup to reduce potential electron or ion scattering losses. Subsequent experiments demonstrated that electron and/or ion scattering loss for the elements studied were $<5 \%$, suggesting the cup geometry was unnecessary because high implant yields were obtained using a thin $(0.05 \mathrm{~mm})$ planar copper foil $(30 \mathrm{~mm} \times 24 \mathrm{~mm})$ placed over the cup aperture. The use of the planar foil geometry simplified chemical recovery of the enriched isotope and reduced potential isotopic contamination by minimizing the weight of the substrate. Laser ablation imaging subsequently also confirmed the implant region was well defined and centrally located within the dimensions of the cup aperture allowing the use of an even smaller implant foil with dimensions of $10 \mathrm{~mm} \times 10 \mathrm{~mm}$. As a result all the implant experiments reported were conducted using electrically conductive adhesive backed copper foil (1181 3/4X18,3M/Interconnect Solutions, USA), that is 
normally used for general radio frequency screening applications. The adhesive backing also serves to hold the collection foil in place in the vertical collector orientation. The presence of rare earths including Eu were detected in the copper foil by LA-ICP-MS, but the low background levels (low ng gram $^{-1}$ ) contributed only a minute level of background isotopic contamination.

Preceding implantation, the surface of the copper foil was cleaned with a mildly abrasive cleaning pad, thoroughly rinsed in a flow stream of $18 \mathrm{M} \Omega$ deionized water, rinsed with high purity isopropyl alcohol and finally dried in a flow stream of argon or nitrogen gas. The foil was then cut to length and mounted on the implant assembly before being inserted into the ICPMS. No degradation of the base $\left(<1 \times 10^{-7}\right.$ mbar $)$ or operating $\left(\approx 8 \times 10^{-7}\right.$ mbar) pressures of the ICP-MS was observed using these materials. Electrical connection to the implant was made using one of the three existing high voltage coaxial feed-thrus normally used to supply the multiplier voltages. Figure 1 is a photograph of the prototype collector assembly with a copper foil affixed to the collector. Once inserted into the vacuum chamber the implant foil was located within $1 \mathrm{~mm}$ of the quadrupole exit lens aperture $(6.0 \mathrm{~mm}$ diameter) to minimize the effects of ion beam spread. Other modifications included widening the bias range of the quadrupole exit lens to $\pm 500 \mathrm{~V}$ and relocating the multiplier preamp board that has to remain attached to the internal instrument controller for the ICP-MS software to operate mass control of the quadrupole normally. The quadrupole peak width was set to standard instrument settings of 0.8 amu at $5 \%$ peak height, that yields an abundance sensitivity of $<1 \mathrm{ppm}$ at $\mathrm{M}-1$ and $<0.5 \mathrm{ppm}$ at $\mathrm{M}+1$. Typical quadrupole pole bias settings were in the range 0 to $-2 \mathrm{~V}$. When operating the isotope collecting foil at ground potential, ion implant current measurements were made using a model 6517B electrometer (Keithley Instruments Inc., Cleveland, OH, USA).

\section{Implant Operation}

All elemental solutions employed in this work were prepared from high purity single element 1000 ppm concentration standards (Inorganic Ventures, Christiansburg, VA, USA). These elemental standards are used in routine elemental analysis and all display natural isotopic abundances. All standard dilutions were prepared by weight using a $2 \% \mathrm{HNO}_{3}$ diluent prepared from 


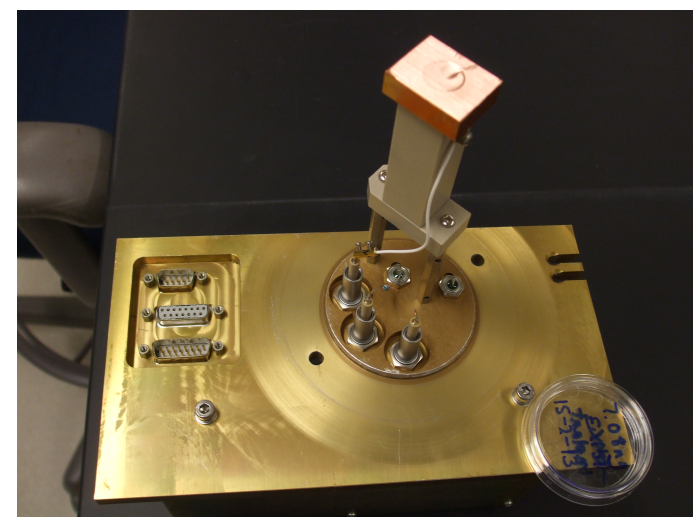

Figure 1: A photograph of the prototype ion collector assembly mounted on the ICP-MS detector flange.

high purity concentrated $\mathrm{HNO}_{3}$ (Optima Grade, Fisher Scientific, Canada) and high purity $18.2 \mathrm{M} \Omega$ deionized water.

Preliminary implant measurements were conducted using $10 \mathrm{ml}$ of a 10 ppm solution of Eu prepared as described previously. The Eu solution was aspirated in the ICP-MS using the standard liquid sample introduction system, comprising a concentric nebulizer and a Peltier cooled impact bead spray chamber held at $3{ }^{\circ} \mathrm{C}$. The ICP operating power was set to $1350 \mathrm{~W}$. As the sample introduction system displays a relatively low efficiency with only $\approx 0.02 \mathrm{~g} \mathrm{~min}^{-1}$ of solution actually entering the plasma with an uptake rate of $0.3 \mathrm{ml} \mathrm{min}^{-1}$ the waste can be effectively recycled without any loss in analyte concentration, ensuring several hours of continuous operation consuming only small volumes $(5-10 \mathrm{ml}$ ) of solution. Re-cycling the solution in this way improves overall efficiency. High efficiency nebulization methods are widely available that employ more aggressive desolvation methods, but the solution sample can only be passed once through these systems as the waste produced is analyte depleted $\approx 10 \%$ compared to the input stock concentration.

Measured collector-implant noise current with the ICP-MS in operate state and the quadrupole mass set to $220 \mathrm{amu}$ was $<+0.05 \mathrm{pA}$. When set to the mass of ${ }^{151} \mathrm{Eu}$.the implant ion current attained a maximum steadystate value of $+160 \mathrm{pA}$ after some minor re-optimization of the ion lenses. In general, maximum ion current was achieved with plasma conditions and ion lens settings very similar to those employed for routine high sensitivity analysis using the electron multiplier. It was observed that the quadrupole exit lens yielded maximum ion current with a bias voltage of $-30 \mathrm{~V}$, and ion 
current was effectively reduced to background noise levels if the exit lens bias voltage was set to $+20 \mathrm{~V}$, indicating the typical ion energy of most ions exiting the quadrupole is $<+10 \mathrm{eV}$. Based on the measured implant current, the average number of incident ${ }^{151} \mathrm{Eu}$ ions was calculated to be: $1.60 \times 10^{-10} / 1.602 \times 10^{-19}$ (electron charge) that equals $9.99 \times 10^{8}$ ions $\mathrm{s}^{-1}$. When the incident ion flux is normalized for the natural abundance of ${ }^{151} \mathrm{Eu}$ and to a solution concentration of $1 \mathrm{ppm}$, there was good agreement with the typical instrument mid-high mass sensitivity observed for a mono-isotopic element with the standard electron multiplier ion detection system of $\approx 2 \times 10^{8}$ $\mathrm{cps} \mathrm{ppm}^{-1}$. The expected accumulation rate of ${ }^{151} \mathrm{Eu}$ would then be given by: $\left(\mathrm{I}_{S} \times \mathrm{A}_{R}\right) / \mathrm{N}_{A}$. Where $\mathrm{I}_{S}$ is the number of incident ions per second, $\mathrm{A}_{R}$ is the Relative Atomic Weight and $\mathrm{N}_{A}$ is the Avogadro Constant. For an ${ }^{151} \mathrm{Eu}$ ion current of $160 \mathrm{pA}$ this equates to a depostion rate of $2.5 \times 10^{-13} \mathrm{~g}$ $\mathrm{s}^{-1}$, or $0.9 \mathrm{ng} \mathrm{hr}^{-1}$ from the input solution concentration of $10 \mathrm{ppm}$.

The implant incident ion current response was measured over a range of elemental concentrations (0-1000 ppm) and for a range of elements at very low and mid - high mass regions. Figure 2 shows the ion current response for $\mathrm{a})^{10} \mathrm{~B}$ and $\left.\mathrm{b}\right)^{151} \mathrm{Eu}$ as a function of elemental concentration, the ${ }^{10} \mathrm{~B}$ ion current response differs quite markedly from that of ${ }^{151} \mathrm{Eu}$. The ${ }^{10} \mathrm{~B}$ ion current response remained linear up to $1000 \mathrm{ppm}$ concentration, although the magnitude of the ion current is about an order of magnitude lower than that observed for ${ }^{151} \mathrm{Eu}$. In part this can be explained by the lower abundance of ${ }^{10} \mathrm{~B}(19.8 \%)$, its relatively high ionization potential and the reduced sensitivity displayed by ICP-MS towards low-mass elements caused by the processes of collisional scattering and space charge repulsion in the plasma sampling interface and ion optics before the quadrupole. As a result even using a solution containing $1000 \mathrm{ppm} \mathrm{B}$, the deposition rate would only be $\approx 0.16$ ng hour ${ }^{-1}$ for ${ }^{10} \mathrm{~B}$. Aspirating more complex mixtures of different elements affects the onset at which non-linearity in the current response versus concentration occurs, particularly for low mass elements, but describing these effects is outside the primary focus of this particular publication. ${ }^{151} \mathrm{Eu}$ ion current increases linearly with concentration up to $100 \mathrm{ppm}$, but then gradually tapers off and does not reach an asymptotic value at $1000 \mathrm{ppm}$. The current linearity limit for ${ }^{151} \mathrm{Eu}$ was in the range $1-2 \mathrm{nA}$, although at 1000 ppm the ion current reached $5.8 \mathrm{nA}$, which equates to a deposition rate of $32.7 \mathrm{ng} \mathrm{hr}{ }^{-1}$. Increased Eu ion currents up to $10 \mathrm{nA}$ were obtained by retuning the ion optics under the higher ion current conditions, offsetting some of the space charge effects responsible for limiting the ion current. 

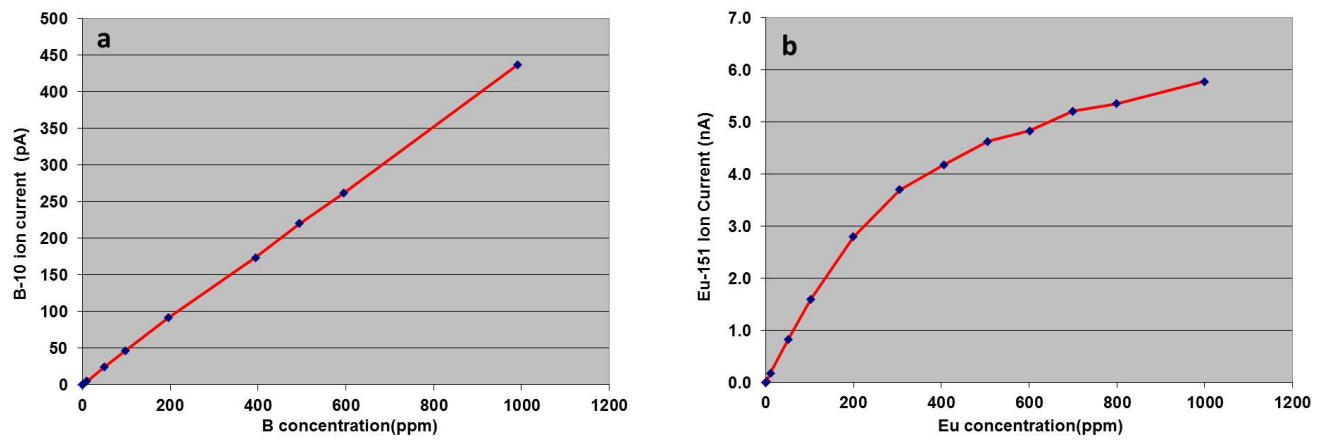

Figure 2: Incident ion current response for (a) ${ }^{10} \mathrm{~B}$ and (b) ${ }^{151} \mathrm{Eu}$ as a function of element input concentration (ppm).

\section{Experimental Results}

\subsection{Imaging the isotopically enriched ${ }^{151} \mathrm{Eu}$}

The first experiment undertaken was to establish that a high degree of isotopic isolation could be achieved by quadrupole ICP-MS on a simple copper foil target. During this first experimental run with a clean copper foil substrate ${ }^{151} \mathrm{Eu}$ was implanted from a solution of $10 \mathrm{ppm}$ Eu for 250 minutes. The total accumulated implant charge measured by the electrometer over this period was $1.40 \times 10^{-6} \mathrm{C}$, which equates to a nominal mass of $2.19 \mathrm{ng}$ of ${ }^{151} \mathrm{Eu}$. The ICP-MS was switched into standby state, followed by venting to remove the implant assembly and the copper foil target. The implanted target was transferred to a laser ablation cell with the intention of imaging the distributions of both the ${ }^{151} \mathrm{Eu}$ and ${ }^{153} \mathrm{Eu}$ isotopes. The ablation was performed using a model 266Macro (Merchantek Inc., CA, USA) coupled to a model X2 quadrupole ICP-MS (ThermoFisher Inc., Winsford, England). Laser wavelength was $266 \mathrm{~nm}$, ablation fluence of $4.5 \mathrm{~J} \mathrm{~cm}^{-2}$, repetition rate $10 \mathrm{~Hz}$, spot size $100 \mu \mathrm{m}$ and scan speed $50 \mu \mathrm{m} \mathrm{s}^{-1}$. The laser was set to ablate across the implant as a series (25) of horizontal line scans $24 \mathrm{~mm}$ long with a line spacing of $500 \mu \mathrm{m}$, while the ICP-MS recorded the response at masses 151 and 153 in time resolved analysis mode. The ICP-MS data was exported into Microsoft Excel and compiled into a three dimensional x-y plot with the analyte intensity plotted on the vertical axis.

Figure 3 shows the spatial distribution of the quadrupole implant of ${ }^{151} \mathrm{Eu}$ on the copper foil and figure 4 the corresponding image for ${ }^{153} \mathrm{Eu}$. Compar- 
ing the distribution of both isotopes it is clear that ${ }^{153} \mathrm{Eu}$ was very effectively rejected with only trace level counts detected for this isotope; the level observed is consistent with natural Eu background in the copper implant substrate with no discernible structure to its distribution. A single ${ }^{153} \mathrm{Eu}$ inclusion with a maximum intensity of 400 counts was detected, but this is the typically observed behaviour for trace level elements that are not spread through a sample homogeneously when using laser ablation. The far higher level of ${ }^{151} \mathrm{Eu}$ counts $\left(\approx 1.25 \times 10^{5} \mathrm{cps}\right.$ at peak) confirms only the target isotope was implanted. The implant ${ }^{151} \mathrm{Eu} /{ }^{153} \mathrm{Eu}$ ratio based on total counts (12094177/2928) accumulated in the main ablated area was 4130, suggesting the ${ }^{151} \mathrm{Eu}$ isotopic purity is now $99.976 \%$, as compared with the natural ${ }^{151} \mathrm{Eu} /{ }^{153} \mathrm{Eu}$ isotopic ratio of the starting material of 0.916 . For comparison, the isotopic distribution of the starting solution (10 ppm natural Eu) used to prepare the implant was ${ }^{151} \mathrm{Eu} 47.81 \%$ and for ${ }^{153} \mathrm{Eu} 52.19 \%$. Had the accumulation time been extended this would have continued to enhance the ${ }^{151} \mathrm{Eu}$ isotopic purity. The shape of the ${ }^{151} \mathrm{Eu}$ distribution was rather odd, forming an asymmetric bimodal pattern that may be related to the ion beam being horizontally pinched by the asymmetric focusing of the deflection optics just prior to entering the quadrupole. Other researchers imaging ion beams exiting from a quadrupole have reported more star-like distributions that align more closely with the open central axis of the quadrupole and the four gaps between the rods $[13,14]$. The other possibility highlighted in the work by Short et al.[15] demonstrated elongation of the exit beam could be caused by ions entering the quadrupole slightly off axis.

\section{2. ${ }^{151}$ Eu implant yield versus accumulated charge}

One initial concern with the simple planar foil implant geometry and low energy ions was possible loss of the target isotope ${ }^{151} \mathrm{Eu}$ atoms caused by atoms failing to attach to the implant substrate, despite being neutralized during the initial impact with the foil. Hu and Houk [9] possibly oberved this effect for low energy ${ }^{165}$ Ho ions being implanted onto a graphite target in their ICP-MS experiments. In addition, as no bias was applied to the implants to minimize any measurement errors induced by bias current leakage, it would not be unreasonable to suspect that ${ }^{151} \mathrm{Eu}$ atom attachment to the foil could be both inefficient and weak. As a result while the electrometer would register the neutralizing charge transfer to the incident ion, the measured total charge would over estimate the total ${ }^{151} \mathrm{Eu}$ atoms accumulated during the implant process. To test the efficiency of this process a series of 


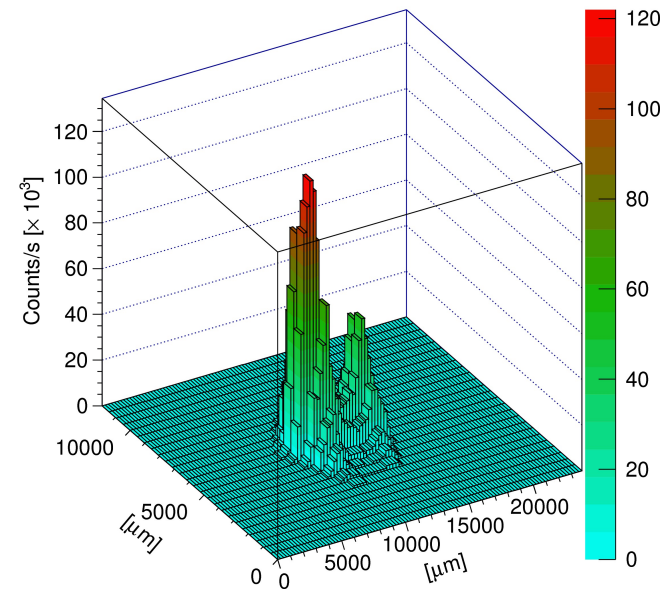

Figure 3: The laser ablation ICP-MS image of ${ }^{151} \mathrm{Eu}$ on the copper foil after implantation.

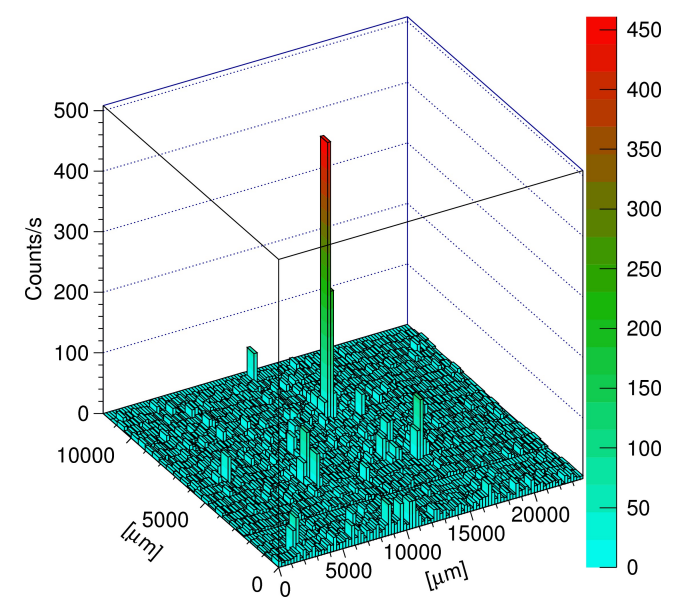

Figure 4: The corresponding laser ablation ICP-MS image of ${ }^{153} \mathrm{Eu}$ on the copper foil after implantation of only ${ }^{151} \mathrm{Eu}$. 
implants were prepared for which the total ${ }^{151} \mathrm{Eu}$ accumulated charge deposited on each was accurately measured and the predicted weight of ${ }^{151} \mathrm{Eu}$ calculated from the total accumulated charge. The implants were then dissolved and analyzed by isotope dilution ICP-MS to measure the actual mass of ${ }^{151} \mathrm{Eu}$ deposited on the copper foil target.

After preparing three implants with differing amounts of ${ }^{151} \mathrm{Eu}$ ranging from 2.7-108 ng, estimated from accumulated charge measurement, the adhesive backing on each foil was carefully removed using a cotton swab soaked in iso-propyl alcohol, followed by rinsing in $18.2 \mathrm{M} \Omega$ deionized water. The central $11 \mathrm{~mm}$ diameter of each copper foil that encompasses the implanted area was removed, taking care not to touch the implanted surface during the process. Each cut out foil ( $\approx 20 \mathrm{mg}$ ) was accurately weighed to $0.01 \mathrm{mg}$ resolution and dissolved in $4 \mathrm{ml}$ of $50 \% \mathrm{v} / \mathrm{v}$ high purity $\mathrm{HNO}_{3}$ in separate cleaned and tared test tubes. Following dissolution the copper solutions were diluted up to a volume of $10 \mathrm{ml}$ using $18.2 \mathrm{M} \Omega$ deionized water and the total weight of each sample stock solution recorded. The ${ }^{151} \mathrm{Eu} /{ }^{153} \mathrm{Eu}$ ratio of all three implant solutions were analyzed both un-spiked and spiked with natural $\mathrm{Eu}\left({ }^{151} \mathrm{Eu} /{ }^{153} \mathrm{Eu}=0.915\right)$. The level of the Eu spike added to each of the three solutions was set to match the expected ${ }^{151} \mathrm{Eu}$ level from each of the respective implant solutions after further dilution to reduce the copper concentration to $5 \mathrm{ppm}$ or $10 \mathrm{ppm}$. Indium was also added as an internal standard. The results of the isotope dilution experiments are summarized in Table 1. The ${ }^{151} \mathrm{Eu}$ yield results suggest that for the combination of Eu with a the copper foil target, implant efficiency is high and measurement of the total charge accumulated is a reliable guide to the weight of the purified isotope attached to the foil target.

\subsection{Study of adjacent mass leakage using Er isotopes}

Contamination of the target enriched isotope with those from adjacent masses is of particular concern in mass spectrometry as these isotopic impurities can limit spiking levels and reduce measurement accuracy. Eu has two natural occurring isotopes that are separated by two mass units, therefore contamination mainly originates from trace levels of natural Eu in the copper foil target. To study possible isotopic leakage on adjacent masses i.e. $\mathrm{m}-1$ and $\mathrm{m}+1$, the element Er was selected for implant experiments where the isotope ${ }^{167} \mathrm{Er}$ was implanted on the copper foil target with the intention of rejecting the adjacent Er isotopes ${ }^{166} \mathrm{Er}$ and ${ }^{168} \mathrm{Er}$. All three isotopes display broadly similar natural abundances $\left({ }^{166} \operatorname{Er}(33.50 \%),{ }^{167} \operatorname{Er}(22.87 \%)\right.$ and 
Table 1: A comparison between ${ }^{151} \mathrm{Eu}$ implant weights estimated from total charge measurement versus \% yield estimated by isotope dilution (ID) ICP-MS.

\begin{tabular}{|c|c|c|}
\hline Implant & $\begin{array}{c}151 \text { Eu Implanted } \\
\text { (ng) deposited by charge }\end{array}$ & \% Yield measured ID \\
\hline 1 & 2.762 & 94.0 \\
\hline 2 & 7.08 & 110.0 \\
\hline 3 & 108 & 98.6 \\
\hline & Average & 100.8 \\
\hline & SD & 8.23 \\
\hline & \% Rsd & 8.16 \\
\hline
\end{tabular}

$\left.{ }^{168} \operatorname{Er}(26.97 \%)\right)$, making this a good selectivity demonstration of the isotopic purification potential of this approach.

A solution concentration of $10 \mathrm{ppm}$ Er with a natural isotopic distribution was employed for the implant experiments performed under similar ICPMS operating conditions used for the Eu implants. Quadrupole mass peak width was $0.8 \mathrm{amu}$. The measured ${ }^{167} \mathrm{Er}$ ion current was $\approx 95 \mathrm{pA}$ and after 217 minutes the total charge accumulated was $1.01 \mu \mathrm{C}$, that equates to a theoretical mass of $1.75 \mathrm{ng}$ implanted. The ICP-MS was vented and the copper foil target removed from the mount and placed in the ablation cell of the LA-ICP-MS system. An area of $14.78 \mathrm{~mm}$ x $14.72 \mathrm{~mm}$ of the copper foil target was ablated during the imaging experiments comprised of 60 horizontal ablation lines. Laser spot size was set to $240 \mu \mathrm{m}$, laser ablation fluence to 5 $\mathrm{J} \mathrm{cm}^{-2}$, line spacing $250 \mu \mathrm{m}$, laser firing rate $5 \mathrm{~Hz}$ with a laser scan rate of $100 \mu \mathrm{m} \mathrm{s}^{-1}$. The ICP-MS responses for the isotopes ${ }^{166} \mathrm{Er},{ }^{167} \mathrm{Er}$ and ${ }^{168} \mathrm{Er}$ were recorded in time resolved analysis mode with a dwell period of 0.5 seconds/isotope during the ablation.

Figures 5, 6 and 7 shows the imaged $\mathrm{Er}$ isotopic distributions on the copper foil target for ${ }^{166} \mathrm{Er},{ }^{167} \mathrm{Er}$ and ${ }^{168} \mathrm{Er}$ respectively. There is no discernible distribution for the Er isotopes ${ }^{166} \mathrm{Er}$ and ${ }^{168} \mathrm{Er}$ on the copper foil target, indicating that both isotopes are very effectively rejected as ions and that there is no sign of these isotopes leaking through the mass spectrometer as neutral atoms co-depositing on the target. The ICP-MS does register some single point spikes for the rejected Er isotopes they are of low intensity $<500$ cps maximum and randomly distributed, with an average background of $<10$ cps indicating trace contamination of the copper with natural Er. For the selected isotope ${ }^{167} \mathrm{Er}$, the distribution observed was very similar to that 
shown earlier for the ${ }^{151} \mathrm{Eu}$ implant, forming an asymmetric bimodal distribution reaching a peak intensity of 300,000 cps, clearly demonstrating that highly selective isotopic enrichment was achieved. The natural isotope ratios for $\operatorname{Er}$ are ${ }^{167} \mathrm{Er} /{ }^{166} \mathrm{Er}=0.681$ and for ${ }^{167} \mathrm{Er} /{ }^{168} \mathrm{Er}=0.854$, in comparison with implant Er ratios of ${ }^{167} \mathrm{Er} /{ }^{166} \mathrm{Er}=1682$ and ${ }^{167} \mathrm{Er} /{ }^{168} \mathrm{Er}=2139$.

\section{Discussion}

The experimental results presented demonstrate that micro scale isotopic purification can be readily accomplished using moderately sensitive quadrupole ICP-MS instrumentation. Such equipment is widely available in any analytical laboratory likely to be performing elemental analysis and isotope ratio measurements by mass spectrometry. The modifications required to the equipment are simple and the source materials readily available in the form of solution concentration standards with natural isotopic distributions for most elements. Even the use of the source material in a solid form is possible using laser ablation and, while not reported in this paper, ion current measurements have been successfully conducted for specific isotopes using that method of sample introduction. This flexibility in sample introduction methods for most elements allowed by using the ICP ion source offers considerable benefits over the Calutron or gas diffusion-centrifuge approaches. For Calutrons the chemical form, even the methods of atomization and ionization vary according the the element being processed. While for gas diffusion-centrifuge approaches the element feed must be converted into a gaseous form that is stable at modest temperatures. The primary limits on the ICP-MS method are set by the space-charge limit of the ion optics and instrument sensitivity versus mass response, that favors mid and high masses over low masses ( $<40 \mathrm{amu})$. Even so, the ICP-MS employed in this work displayed a linear response for the exit ion beam of the quadrupole up to $2 \mathrm{nA}$. That would equate to deposition rates for mid-high mass elements of 6-18 ng hour $^{-1}$. Potential deposition rates up to 90 ng hour ${ }^{-1}$ would be possible by operating with ion currents of $10 \mathrm{nA}$ where the quadrupole exit ion current becomes non-linear with respect to input concentration. However, sustained operation might become difficult, with problems caused by gradual blocking of the skimmer aperture in the plasma-sampling interface. Operating at these higher ion currents might reduce implant efficiency through sputtering losses, although this could be overcome by adopting the angled pocket collector design adopted on Calutrons [16, 17]. There may also be an increase 


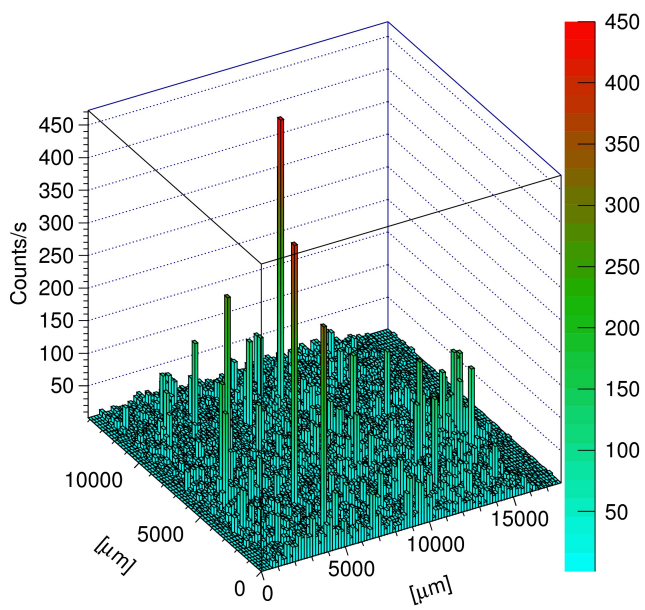

Figure 5: The laser ablation ICP-MS image of ${ }^{166} \mathrm{Er}$ on the copper foil after implantation of only ${ }^{167} \mathrm{Er}$.

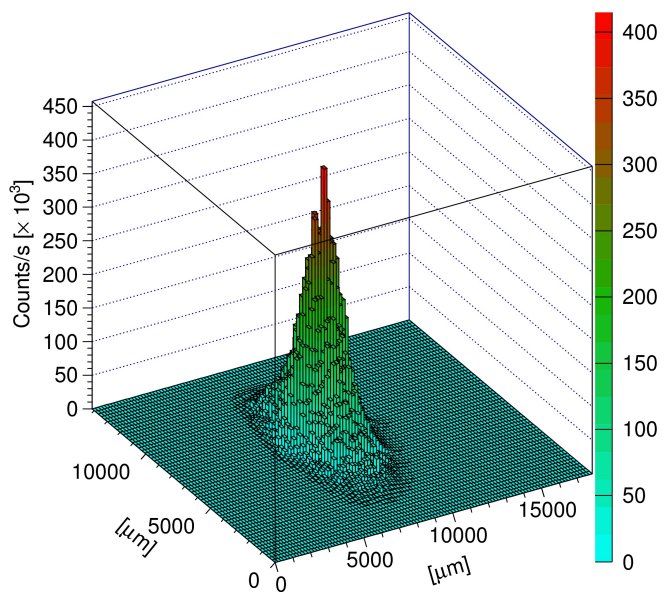

Figure 6: The laser ablation ICP-MS image of ${ }^{167} \mathrm{Er}$ on the copper foil after implantion. 


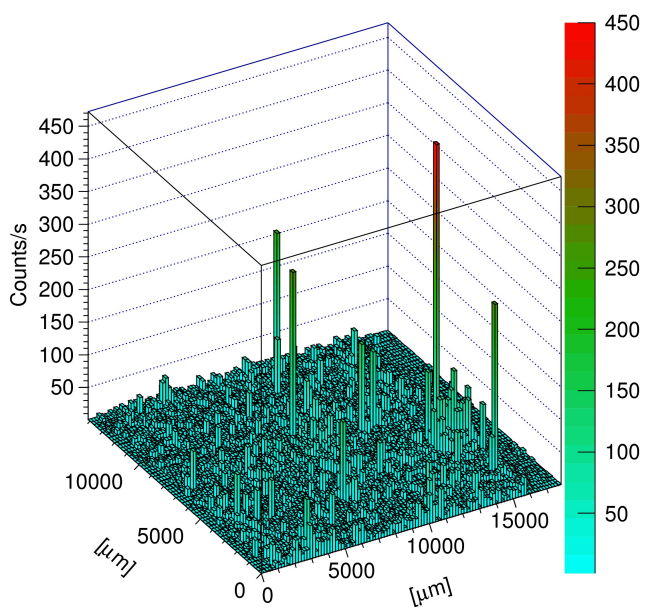

Figure 7: The laser ablation ICP-MS image of ${ }^{168} \mathrm{Er}$ on the copper foil after implantation of only ${ }^{167} \mathrm{Er}$.

in the probability of either neutral atoms or ions of unwanted isotopes being co-implanted with the target isotope. These possibly adverse high ion current effects would require further investigation.

Careful measurements of low energy ${ }^{151} \mathrm{Eu}$ ion deposition onto a copper foil demonstrated vitually $100 \%$ ion collection efficiency. This maybe a result of the fortuitous combination of $\mathrm{Eu}$ on $\mathrm{Cu}$, as other workers [9] studying ${ }^{165} \mathrm{Ho}$ ion deposition on graphite observed lower deposition rates for low energy ${ }^{165} \mathrm{Ho}$ ions, compared to those with higher ion energies. Actual Ho ion deposition efficiency could not be estimated as the incident ${ }^{165} \mathrm{Ho}$ ion current was not measured. However the literature results highlight that deposition efficiency for ions of different elements maybe quite variable depending on the composition of the implant substrate and ion energy conditions under which implantation takes place. At high ion energies sputtering of deposited material by incoming ions must also be considered as a possible factor in the reduction of ion collection efficiency.

The quadrupole ICP-MS modified for this study dates from the late 1990s, displaying an ion transmission efficiency of $\approx 0.007 \%$ for mid-high mass elements, where ICP-MS tends to display the highest sensitivity. Some of the latest bench top ICP-MS instruments display improved efficiencies of $\approx 0.035 \%$. While this might seem very poor, the standard aqueous sample introduction systems found on most instruments allow the stock solution to 
be recycled to exhaustion. So, a $10 \mathrm{ml}$ solution can be made to last $6-8$ hours because the amount of aerosol entering the plasma is only $0.02-0.03 \mathrm{~g} \mathrm{~min}^{-1}$. This effectively allows a 10-fold increase in the amount of an isotope that can be deposited from the same volume compared to only using a single solution pass.

During these initial studies the deposition area of the enriched isotope was fairly large ( $\approx 6 \mathrm{~mm} \times 6 \mathrm{~mm}$ ) with little obvious focusing observed by varying the bias voltage on the exit lens. Shrinking the deposition area would improve isotopic purity as it would require less of the implant substrate to be processed to recover the purified isotope. While the quadrupole in this spectrometer has rf-only pre-filters, there are no rf-only post filters that could reduce exit aperature divergence in the ion beam that in turn might shrink the deposition area. One could reduce the aperture of the quadrupole exit lens, currently $6 \mathrm{~mm}$ in diameter, although this may reduce the ion current hitting the implant. Sufficient space is available within the detector chamber to add additional ion optics to focus the ion beam onto the implant surface. While neither of these potential improvements have been investigated yet, experiments applying a negative bias to the implant have shown the implant area can be reduced, but at the expense of making measurement of the ion current and accumulated charge more difficult, as a result of bias voltage leakage.

All the results reported here are focused on enrichment and implantation of one single isotope of an element at a time, as this highlights the single mass limitation of the quadrupole mass filter. The possibility exists of implanting multiple isotopes of an element to create a tailored isotopic distribution suitable for some specific application like mass bias correction in isotope ratio measurements. For a quadrupole based ICP-MS this is probably best performed sequentially, one mass at a time. While mass peak jumping is easily programmed through the software to cover multiple isotopes, if this involves skipping over unwanted isotopes slight transmission of these isotopes will occur scanning both up and back down the mass range, as there is no gatingoff of the ion beam during this process in commercial ICP-MS instruments. However, this feature could easily be added by applying a positve high voltage bias to either a lens or the quadrupole on an instrument designed for this application. The use of magnetic sector multicollector ICP-MS is a possibility to selectively collect multiple isotopes of an element simultaneously, however these remain considerably more expensive and therefore less readily available than quadrupole based ICP-MS. In addition the isotopic purity ob- 
Table 2: Certified isotopic distribution of Pu in CRM-131 in 1981.

\begin{tabular}{|c|c|c|c|c|c|c|}
\hline Pu Isotope & 238 & 239 & 240 & 241 & 242 & 244 \\
\hline Atom \% & 0.005 & 0.034 & 0.677 & 0.092 & 1.325 & 97.867 \\
\hline
\end{tabular}

tained from a magnetic sector maybe slightly inferior owing to their usually poorer abundance sensitivity performance compared to quadrupole based systems.

Isotopic purification of some radioactive tracers is another area where this approach could make a significant impact. For example ${ }^{244} \mathrm{Pu}$ is widely used in the quantification of ${ }^{239} \mathrm{Pu}$ and ${ }^{240} \mathrm{Pu}$, but all ${ }^{244} \mathrm{Pu}$ available contains significant traces of all the $\mathrm{Pu}$ isotopes from ${ }^{238} \mathrm{Pu}$ to ${ }^{242} \mathrm{Pu}$. Table 2 highlights the isotopic distribution of this certified reference material CRM-131 (New Brunswick Laboratory Argonne, IL, USA). By applying this isotopic purification approach it should be possible to achieve ${ }^{244} \mathrm{Pu}$ isotopic purity $>99.999 \%$, greatly reducing the atom $\%$ of all the lower mass isotopes given their mass spacing from the target ${ }^{244} \mathrm{Pu}$ and the additional benefit gained from there being no natural background for this element in the implant substrate. That should make recovery of the highly purified ${ }^{244} \mathrm{Pu}$ very straight-forward. This process could also be applied to other currently unobtainable rare isotopes like ${ }^{236} \mathrm{~Np}$, that may be present at low levels in some ${ }^{237} \mathrm{~Np}$ waste streams produced during nuclear fuel reprocessing, as no other long-lived isotopic tracer for accurate quantification of ${ }^{237} \mathrm{~Np}$ in environmental samples exists.

One final benefit of this isotope selective method that has been described is its application to improving radiation measurements [18]. As mass spectrometry is inherently selective, the technique can effectively be used as a sample processing tool to isolate some target isotope from unwanted interferences that may also be present in a sample. Conventionally, this would require wet chemistry separations, but using ICP-MS as the sample processing tool it should be possible to prepare implants with the target isotope largely isolated from a sample matrix. The sample could be presented in either liquid or solid form. While quantification would be challenging, using this approach it may be adequate for rapid screening applications where the presence of a particular isotope may target that sample for further more rigorous analysis. For $\alpha$-spectrometry we used the ICP-MS to implant trace levels of ${ }^{241} \mathrm{Am}$ and compared the $\alpha$-energy spectrum with those produced by the standard methods of -sample preparation of electro-deposition and direct solution stippling $[19,20,21]$. The ICP-MS implanted ${ }^{241} \mathrm{Am} \alpha$-spectrum dis- 
played considerable improvement in energy resolution compared to samples with similar levels of activity produced using the two conventional methods highlighted. This improvement in energy resolution is attributed to the purity and low ion energy surface deposition achieved by ICP-MS, whereas conventional preparation methods partially bury the ${ }^{241} \mathrm{Am}$ atoms with other impurity atoms. It should also be possible to apply this method of sample preparation to ultrathin film sources (nm- $\mu \mathrm{m}$ thick) and to enhance aspects of $\beta$ and $\gamma$ radiation measurements.

\section{Conclusion}

This work shows for the first time that quadrupole ICP-MS can be modified to generate small quantities (ng- $\mu \mathrm{g}$ ) of a single isotope of an element with extremely high isotopic purity (>99.99\%). High rejection of adjacent mass isotopes was readily demonstrated with Er. Collection efficiency for Eu appears to be very high, approaching $100 \%$ even using a simple planar copper foil target, based on accumulated charge-isotope dilution measurements. While overall ion loss from the solution source to the implant substrate are large, equivalent to $\approx 0.005 \%$ ion transmission efficiency, elemental concentration standards are cheap and recycling the waste from the sample introduction system improves efficiency to $0.05-0.5 \%$, at the penalty of extending deposition time. The use of more sensitive ICP-MS instruments that are currently available would improve efficiency to $\approx 1 \%$ or higher. The potential applications of this development should be considerable for mass spectrometric isotope ratio measurements, where highly enriched spikes or tailored isotope distributions could easily be prepared using this widely available bench top instrumentation.

\section{Acknowledgments}

The research described in this paper is part of the Ultrasensitive Nuclear Measurement Initiative at Pacific Northwest National Laboratory. It was conducted under the Laboratory Directed Research and Development Program at PNNL, a multiprogram national laboratory operated by Battelle for the U.S. Department of Energy.

[1] A. O. Nier, E. T. Booth, J. R. Dunning, A. V. Grosse, Nuclear fission of separated uranium isotopes, Phys. Rev. 57 (1940) 546. 
[2] W. E. Parkins, The uranium bomb, the calutron, and the space-charge problem, Phys. Today 58 (5) (2005) 45-51.

[3] R. H. Dyer, D. B. Janney, Operation of the gaseous diffusion enrichment process, in: IEEE Trans. on Power Apparatus and Systems, Vol. 4 of PAS-92, 1973, pp. 1180-1185.

[4] D. R. Olander, The gas centrifuge, Scientific Am. 239 (1978) 37-40.

[5] K. P. Carney, J. J. Horkley, C. A. McGrath, A. J. Edwards, J. E. Davies, G. C. Knighton, J. D. Sommers, J. J. Giglio, Advancement of isotope separation for the production of reference standards, J. Radioanal. Nucl. Chem. 296 (2013) 383-387.

[6] B. J. Egle, K. J. Hart, W. S. Aaron, Stable isotope enrichment capabilities at oak ridge national laboratory, J. Radioanal. Nucl. Chem. 299 (2014) 995-999.

[7] P. S. Mayer, F. Turecek, H. N. Lee, T. N. Scheidemann, A. A. Olney, F. Schumacher, P. Strop, M. Smrcina, M. Patek, D. Schirlin, Preparative separation of mixtures by mass spectrometry, Anal. Chem. 77 (2005) 4378-4384.

[8] A. Bodin, R. Laloo, P. Abelhou, L. Guiraud, S. Gauthier, D. Martrou, An energy-filtering device coupled to a quadrupole mass spectrometer for soft-landing molecular ions on surfaces with controlled energy, Rev. Sci. Inst. 84.

[9] K. Hu, R. S. Houk, Ion deposition by inductively coupled plasma mass spectrometry, J. Vac. Sci. Technol. A 14 (1996) 370-373.

[10] Y.-T. Hsieh, G. M. Henderson, Precise measurement of 228Ra/226Ra ratios and $\mathrm{Ra}$ concentrations in seawater samplea by multi-collector icp mass spectrometry, J. Anal. At. Spectrom. 26 (2011) 1338-1346.

[11] High sensitivity magnetic sector icp-ms sensitivity specification brochure, http://www.thermo.com/eThermo/CMA/PDFs/Product/ productPDF_56336.PDF (2010).

[12] G. R. Gillson, D. J. Douglas, J. E. Fulford, K. W. Halligan, S. D. Tanner, Nonspectroscopic interelement interferences in inductively coupled plasma mass spectrometry, Anal. Chem. 60 (1988) 1472-1474. 
[13] T. E. Kane, V. J. Angelico, V. H. Wysocki, Use of condensation figures to image low-energy ion beam damage to monolayer films, Anal. Chem. 66 (1994) 3733-3736.

[14] Extrel application note gt-712c, extrel cms, 575 epsilon drive, pittsburgh, pa 15238, http://www.extrel.com/Module/ Catalog/CatalogDocFileFile/Quadrupole_Mass_Filters_and_ Ion_Guides/Quadrupole_Mass_Filters/GT-712_Quadrupole_ Emittance_Characteristics/GT-712C_Quadrupole_Emittance_ Characteristics.pdf?id=65 (2005).

[15] R. T. Short, C. C. Grimm, P. J. Todd, Monitor for displaying real-time images of low energy ion beams, J. Am. Soc. Mass Spectrom. 2 (1991) 226-231.

[16] M. L. Smith, Electromagentically Enriched Isotopes and Mass Spectrometry, John Wiley \& Sons, New York, NY, 1956.

[17] J. Koch, R. H. V. M. Dawton, M. L. Smith, W. Walcher, Electromagnetic Isotope Separators and Applications of Electromagentically Enriched Isotopes, Interscience Publishers Inc, New York, NY, 1958.

[18] M. P. Dion, M. Liezers, O. T. Farmer III, B. W. Miller, S. M. Morley, C. J. Barinaga, G. C. Eiden, Improving alpha spectrometry energy resolution by ion implantation with ICP-MS, J. Radioanal. Nucl. Chem. (2014) DOI 10.1007/s10967-014-3500-8.

[19] R. S. Strebin Jr., D. M. Robertson, Alpha-energy and mass analyses of plutonium from a single sample mount, J. Radioanal. Chem. 40 (1977) 213-216.

[20] E. M. Bond, W. A. Moody, D. E. Dry, M. W. Rabin, Factors affecting the quality of plutonium deposits by electrodeposition, J. Radioanal. Nucl. Chem. 296 (2013) 793-796.

[21] V. Jobbagy, M. T. Crespo, R. V. Ammel, M. Marouli, A. Moens, P. S., G. Torano, Preparation of high-resolution U238 alpha sources by electrodeposition: a comprehensive study, J. Radioanal. Nucl. Chem. 298 (2013) 345-352. 
Graphical Abstract Quadrupole Inductive Coupled Plasma - Mass Spectrometer
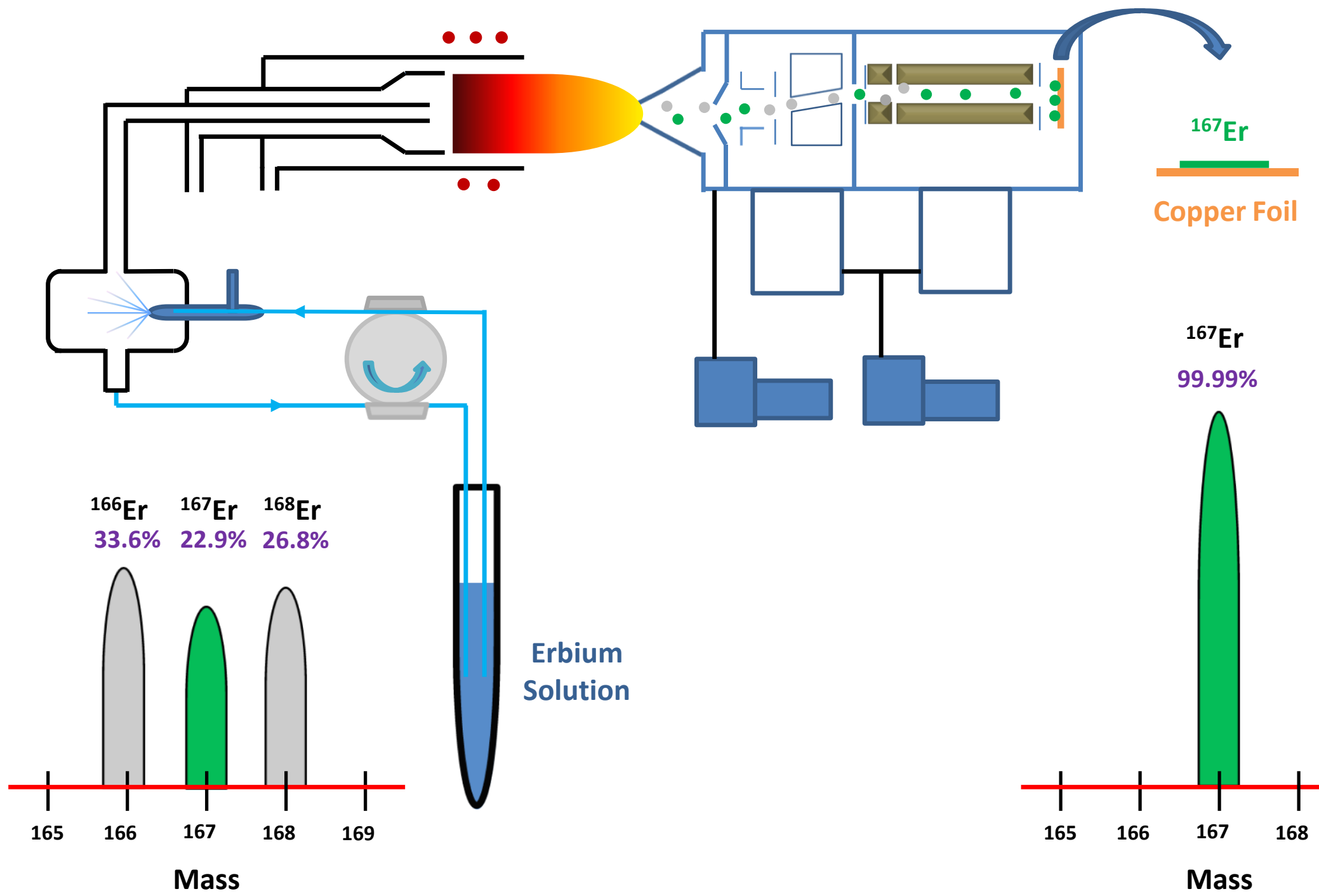

Natural Erbium 